\title{
MODELO PARA PRIORIZAÇÃO DA MANUTENÇÃO PREVENTIVA EM PATRIMÔNIOS HISTÓRICOS EDIFICADOS: CASO DA CIDADE DE SOBRAL-CE
}

\author{
OLIMPIO, LUIZ CARLOS MAGALHÃES \\ Mestrando em Engenharia Civil \\ Universidade Federal do Ceará \\ Ceará, Brazil \\ olimpio@alu.ufc.br
}

\author{
CAMPOS, VANESSA RIBEIRO \\ Professora \\ Universidade Federal do Ceará \\ Ceará, Brazil \\ vanessa.campos@ufc.br
}

\author{
MESQUISTA, ESEQUIEL FERNANDES TEIXEIRA \\ Professor \\ Universidade Federal do Ceará \\ Ceará, Brazil \\ emesquisa@ufc.br
}

\section{RESUMO}

O patrimônio histórico edificado deve ser preservado devido seu inestimável valor tangível e intangível. Neste âmbito a sua manutenção se torna complexa por considerar aspectos não só estéticos e estruturais, mas também subjetivos. Este trabalho apresenta um framework para tomada de decisão multicritério na manutenção preventiva em patrimônios históricos edificados no centro histórico tombado da cidade de Sobral-CE. O modelo coleta o conhecimento de especialistas de áreas chaves para a ponderação dos critérios por meio de método que adota o teorema de Bayes (Bayesian Best-Worst). Nove subcritérios são escolhidos e submetidos a avaliação e estão compreendidos em três critérios nomeados vulnerabilidade estrutural, significância cultural, e potencial e restrições. As alternativas deverão ser avaliadas então em duas etapas: a primeira remete a análise da vulnerabilidade estrutural considerando a sismicidade do município sobralense realizado pelo LAREB e modificado para variáveis fuzzy; a segunda é a coleta de preferências de decisores. Por fim deve ser realizada a aplicação de método de priorização para decisões em grupo (FG-MULTIMOORA) permitindo a ordenação hierárquica. $\mathrm{O}$ trabalho contribui apresentando um modelo de decisão robusto e inédito na manutenção do patrimônio histórico edificado, critérios considerando a vulnerabilidade estrutural, critérios alinhados com as diretrizes de preservação do patrimônio material e ao estudo de tombamento, como também aspectos econômicos e sociais importantes para o município. Este trabalho apresenta resultados preliminares do modelo, que consiste na sua construção, e na seleção e ponderação dos critérios.

Palavras-chave: Patrimônio histórico edificado, Manutenção, Modelo de tomada de decisão.

\section{ABSTRACT}

Built historical heritage must be preserved due its priceless tangible and intangible value. In this context, its maintenance becomes complex because it considers not only aesthetic and structural aspects, but also subjective ones. The decisionmaking process for the use of limited resources for this purpose must take place after a systematic and rational prioritization process. This paper presents a framework for multicriteria decision making in the preventive maintenance of built historical heritage in the historic center of the city of Sobral-CE. The model collects expert knowledge of key areas for criteria weighting using a method that adopts the Bayes theorem (BBWM). Nine sub-criteria are assessed and comprised in three criteria named structural vulnerability, cultural significance, and potential and constraints. The built historical heritage will be evaluated in two stages: The first is the analysis of structural vulnerability considering the seismicity of the city of Sobral, carried out by the Structural Assessment and Rehabilitation Laboratory (LAREB), and modified for fuzzy variables. The second is the collection of decision makers' preferences. Finally, the prioritization method to group decisions will be applied (FG-MULTIMOORA), conceding hierarchical ordering. This paper contributes by presenting a robust and novel decision model for the maintenance of built historical heritage, considering the structural vulnerability, multiple criteria aligned with the material heritage preservation charters and national listing studies, as well as important economic and social aspects for the city. This paper presents preliminary results from the model, consisting of its construction, the selection and weighting of criteria.

Keywords: Built historical heritage, Maintenance, Decision making model. 


\section{INTRODUÇÃO}

A manutenção das edificações é uma problemática que tem ganhado a atenção das organizações responsáveis pelos edificados e profissionais envolvidos com os procedimentos. Isto tem se dado pelo reconhecimento da necessidade de atividades que envolvem a preservação e requalificação dos sistemas que compreendem a edificação de modo a esta atender sua função durante sua vida útil, ainda preservando o conforto e a segurança dos usuários. No contexto de patrimônios históricos edificados as atividades de manutenção devem, também, atender outros aspectos, e é recorrentemente tratada com mais propriedade por fazer parte de planos de conservação que consideram as singularidades destas construções. Ainda, este tema tem destaque devido a contribuição de organizações e instituições nacionais e internacionais na valorização do edificado e no reconhecimento da problemática, e está se tornando progressivamente mais importante ao se conhecer os potenciais benefícios na conservação do patrimônio histórico e cultural para a sociedade (PERNG; JUAN; HSU, 2007).

Os patrimônios costumam passar por procedimentos de tombamento para serem apropriadamente geridos, buscando-se preservar sua significância cultural, sendo o plano de conservação um instrumento da gestão do patrimônio cultural (IPHAN, 2018). Este plano tem o propósito de proteger o bem, permitindo que este seja transmitido à futuras gerações assim como foram de gerações passadas para a presente (KUTUT; ZAVADSKAS; LAZAUSKAS, 2013) e deve envolver o desenvolvimento de estratégias que analisem sua significância ao seu uso, bem como com os objetivos da organização a qual pertence ou ocupa. Tal abordagem deve focar em ações, processos e priorizações buscando a proteção do valor dos patrimônios edificados (BOND; WORTHING, 2016, p.3), não só, o plano deve considerar a preservação de outros sistemas e a estrutura essenciais para a edificação (ICOMOS, 2003; WARREN, 2004).

Deste modo, as ações de manutenção devem proteger o valor e contribuir com a função a qual o patrimônio edificado exerce aos seus usuários, como em IPHAN (2010) que define manutenção como o "conjunto de operações destinadas a manter, principalmente, a edificação em bom funcionamento e uso" e com as atividades que compreendem a conservação em IPHAN (2018), as quais devem preservar os valores de tombamento, estimular a manutenção, otimizar investimentos e reduzir riscos. Gard'ner (2007) complementa quando apontar que também se deve avaliar as vulnerabilidades às quais o patrimônio está submetido, que incluem, mas não se limitam a sua condição física e deterioração, danos e potenciais vandalismos, reparos anteriores inadequados e uso inapropriado. Nesta perspectiva, entende-se a necessidade de avaliar os patrimônios arquitetônicos não só como monumentos, mas também como edificações com fins operacionais, para além da função histórica, cultural e social.

Apesar, observa-se ainda reduzida participação de disciplinas e atores que deveriam ser importantes no processo de decisão na manutenção destes edificados, principalmente no âmbito nacional. As decisões realizadas sem considerar a pluralidade de áreas irão, provavelmente, resultar na redução da significância cultural e do patrimônio de alguma forma (BOND; WORTHING, 2016, p.12). Porém, nesta perspectiva se reconhece a complexidade da decisão devido a multiplicidade de critérios, sobretudo quando tangem a priorização de alternativas frente aos recursos limitados. Para a solução adequada e sistemática destes tipos de problema o ramo da pesquisa operacional desenvolveu os métodos de tomada de decisão multicritério (MCDM). Estes métodos são reconhecidos pela eficácia e objetividade, e são amplamente adotados em problemas complexos (ZAVADSKAS; TURSKIS; KILDIENĖ, 2014). No entanto, a sua aplicação não é frequente na solução de problemáticas envolvendo a preservação de patrimônios históricos edificados (MORKŪNAITE்; KALIBATAS; KALIBATIENE், 2019), e até a presente data não existem publicações nacionais que os adotem nos processos decisórios nestes patrimônios.

Reconhecendo esta lacuna, e identificando a oportunidade de sistematização do processo decisório, este trabalho propõe um modelo de tomada de decisão multicritério na manutenção preventiva de patrimônios edificados. Para isto, se tomou como objeto de estudo o centro histórico tombado da cidade de Sobral-CE, cuja representatividade para a cultura, economia, e história da região e Estado é indiscutível, sendo certamente a cidade que mais resguarda o patrimônio histórico edificado do Ceará (CAMPELO, 2008). O modelo considera os múltiplos critérios importantes para a significância cultural do patrimônio, apontando para a preservação daqueles encontrados no estudo de tombamento em Iphan (1999), nos critérios representativos atualmente, além de oportunidades e vulnerabilidades identificadas em pesquisa. O modelo é construído adotando o conhecimento especializado com dois métodos distintos, o primeiro utilizando o teorema de Bayes para a consolidação e ponderação dos critérios, e o segundo adotando a priorização das edificações selecionadas usando a lógica fuzzy para tratar as incertezas dos decisores. Pretende-se com isto contribuir no processo decisório desta problemática, e trazer para o âmbito de patrimônios históricos uma visão inclusiva das outras disciplinas, de forma objetiva e operacional, podendo ser atribuído ao plano de conservação adotado pelas organizações responsáveis pelos bens, e inclusive ser objeto a ser apreciado no planejamento da cidade. 


\section{MÉTODO E RESULTADOS}

O modelo de tomada de decisão a ser construído para a priorização da manutenção preventiva de patrimônios históricos seguirá algumas etapas, apresentadas na Figura 1. Este modelo envolve a edução do conhecimento de especialistas para alimentar ambos os métodos. Esta estratégia foi preferida pelo fácil acesso as informações devidamente pertinentes para os critérios a serem adotados. Ainda, se vale de informações qualitativas e quantitativas provenientes da pesquisa de Mota, Gadelha e Mesquisa (2017). O modelo se inicia com a etapa de identificação do problema e reconhecimento do cenário. Isto foi realizado com a revisão da literatura e com a pesquisa em campo, que se valeu tanto da análise da vulnerabilidade e na identificação de manifestações patológicas dos edificados históricos, como também com a leitura de documentos e rodas de conversas de agentes relevantes envolvidos com a problemática.

A próxima etapa consiste na ponderação dos critérios, que será realizada através da edução do conhecimento de especialistas. Antes são selecionadas ás áreas das múltiplas disciplinas que compreendem os critérios, para então ser realizada a escolha de especialistas que compreendam o cenário. As informações coletadas são dados de entrada para um método de construção e agregação dos pesos. Posteriormente é realizada a construção da matriz de preferência. Esta etapa consiste primeiramente na coleta dos dados da análise de risco estrutural, e em sua transformação em variáveis fuzzy, considerando a incerteza na construção dos indicadores de vulnerabilidade, um dos critérios considerados nesta pesquisa. Paralelamente os decisores apresentam suas preferências através de funções fuzzy previamente apresentadas e ajudam a construir a matriz de decisão às alternativas. Essa matriz, ponderada pelos pesos dos critérios, e submetidas a um método de decisão resultam na hierarquização das alternativas. A construção dessa matriz não faz parte do produto deste trabalho, que se limita a etapa de planejamento, consolidação e ponderação dos critérios.

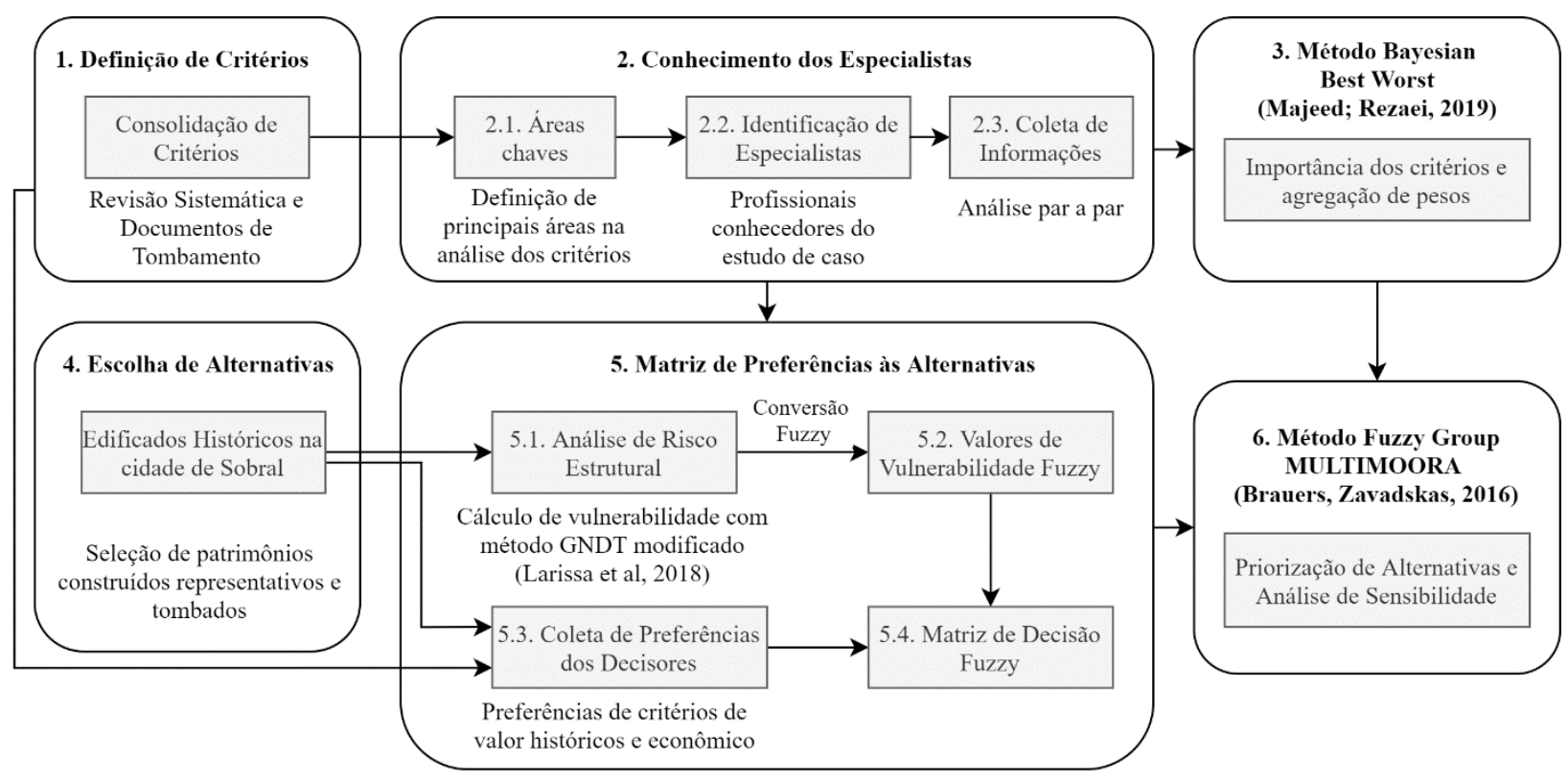

Figura 1: Framework para processo decisório

\subsection{Bayesian Best Worst Method (BBWM)}

Este método foi desenvolvido em Mohammadi e Rezaei (2019), que realizam uma abordagem estatística do método já consolidado nomeado Método Best-Worst, criado e conceitualizado em Rezaei (2015). Os resultados da nova abordagem são semelhantes ao método de origem, entretanto tem uma nova capacidade, a de realizar a agregação de múltiplos especialistas ou decisores, sem a criação de um agente virtual. O novo método, realizado no software Matlab (2018), facilitou a construção de um novo ranking dos critérios, denominado pelos autores de Credal Ranking. O diferencial deste se dá no cálculo da confiança, realizada com base na distribuição Dirichlet, posterior de wagg (vetor de pesos ótimos), enquanto outros métodos de classificação geralmente levam dois números, ou intervalos, e tentam encontrar até que ponto um critério é superior à outro. O Credal Ranking tem uma saída gráfica no software através do weighted directed graph. Os passos do método são apresentados na Figura 2.

Até o terceiro passo o método que adota o teorema de Bayes é similar ao original, ou seja, ambos utilizam os mesmos vetores de entrada $A_{B}$ e $A_{W}$. No quarto passo a função de otimização para a determinação do vetor de pesos dos especialistas difere daquele encontrado no BBWM, que trata os critérios como eventos aleatórios e seus pesos como sua probabilidade de ocorrência por realizar o cálculo do método por meio de inferência estatística. Devido a extensividade, 
não serão apresentados neste trabalho as equações utilizadas no quarto e quinto passo, que podem ser consultadas com detalhes no capítulo 4 e 5 do trabalho de Mohammadi e Rezaei (2019).

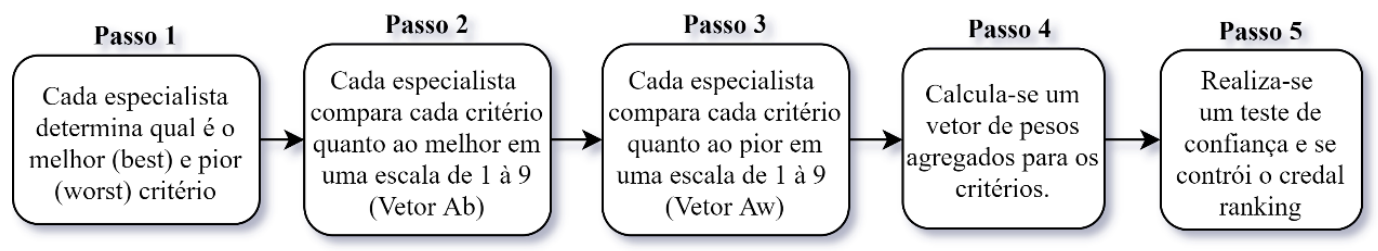

\subsection{Caso}

Figura 2: Passos do método BBWM

O município de Sobral, com 2.122,989 km², implantado a 69 m do nível do mar e distante cerca de 250 km de Fortaleza, situa-se na mesorregião Noroeste e na microrregião Sobral. A cidade teve seu centro histórico tombado em 1999, sendo protegido em nível Federal pelo Decreto-Lei no 25 de 1937 (BRASIL, 1937). Os elementos que ambientam o conjunto urbanístico de Sobral estão contidos na poligonal que delimita o seu entorno, inclusos nesta área marcos visuais importantes em múltiplas disciplinas e para a comunidade.

Para o IPHAN (1999), o que norteou a proteção do núcleo histórico de Sobral foi a oportunidade e necessidade de reconhecê-lo e preservá-lo como um dos mais importantes documentos do urbanismo colonial nesta região, e da história do desbravamento e da ocupação do sertão nordestino. Ainda, mais do que um monumento arquitetônico e urbanístico de valor artístico excepcional, as áreas urbanas contem em seu espaço uma alta concentração de informações históricas relevantes para diversas áreas do conhecimento humano. Com esses atributos, Sobral constitui um interessante estudo de caso para a discussão da contribuição dos processos preservacionistas ao desenvolvimento socioeconômico e humano (DUARTE JUNIOR, 2012, p.214) e justifica sua escolha nesta pesquisa.

\subsection{Seleção de Alternativas}

Composto por cerca de 1.200 imóveis na área de proteção rigorosa, o Centro Histórico de Sobral Dentre as edificações localizadas na área de tombamento, destacam-se 20 edificações marcos na paisagem urbana de Sobral apresentadas e identificadas na Figura 3.

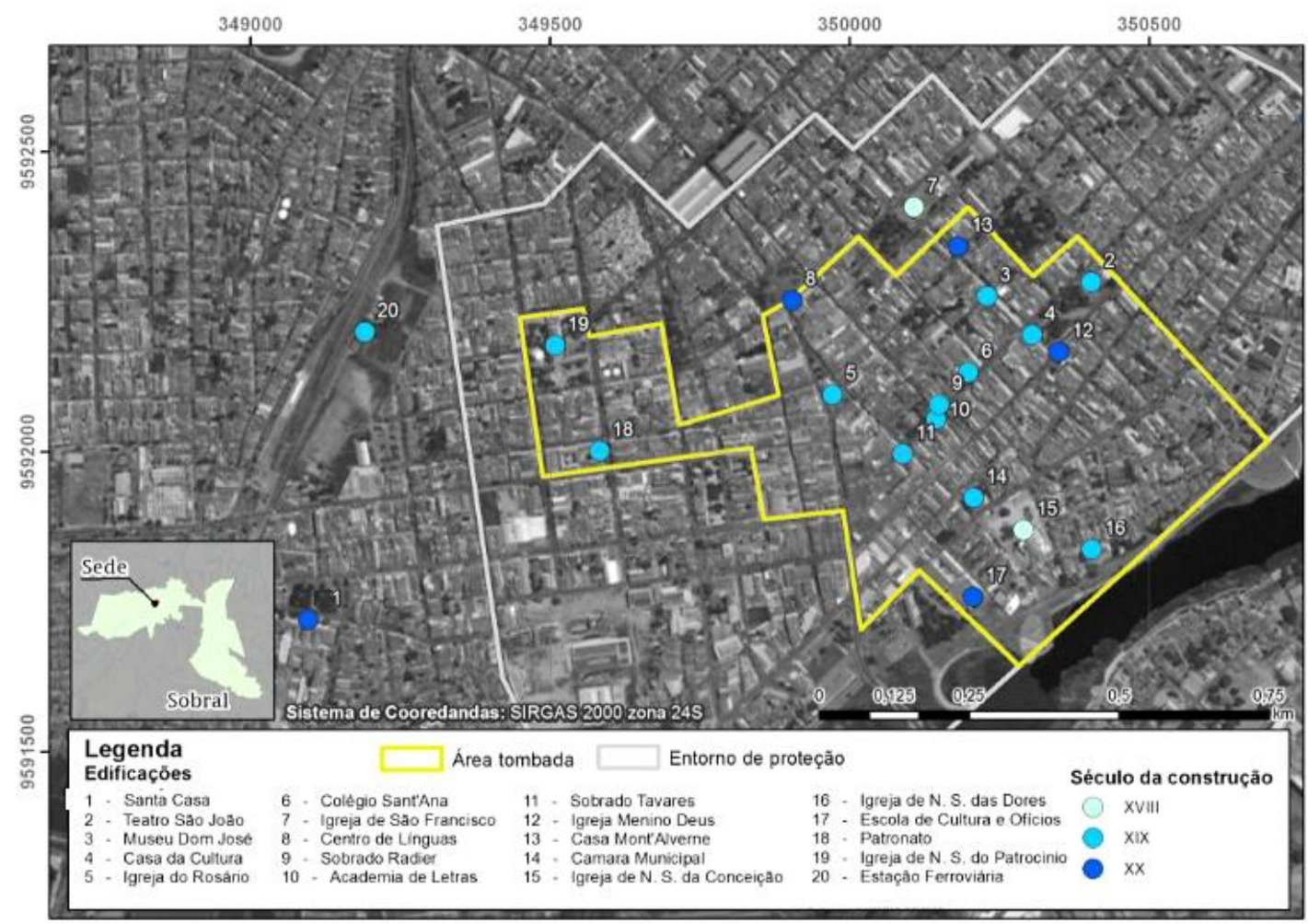

Figura 3: Alternativas 
Elas são essencialmente igrejas, sobrados e solares construídos nos séculos XVIII, XIX e XX, e ilustrarão o funcionamento do modelo neste trabalho. Estas mesmas edificações compuseram, em parte, o estudo de Mota, Gadelha e Mesquisa (2017) na análise de vulnerabilidade estrutural, nos quais estes dados serão usados na hierarquização das alternativas sob este critério. A exceção fora da área de entorno do tombamento são a Santa Casa da Misericórdia (1) e a Estação Ferroviária (20), entretanto são mencionadas como importantes referências arquitetônicas e históricas da cidade no estudo do IPHAN (1999, p.129) e também sugeridos de tombamento estadual por Duarte Júnior (2012).

\subsection{Critérios}

Como definido pelo Icomos (2003) em "princípios da análise, conservação e restauração estrutural do patrimônio arquitetônico" a conservação, reforço e restauração do patrimônio arquitetônico requerem uma abordagem multidisciplinar. Estas múltiplas disciplinas podem ser traduzidas em critérios, sendo a etapa de sua definição uma das mais importantes da construção do modelo decisório. Os critérios devem ser suficientemente capazes de traduzir a função de preferência dos múltiplos especialistas envolvidos no processo, sendo também adequada a não inclusão daqueles pouco importantes, isto pois podem interferir nos resultados e aumentar a complexidade do modelo desnecessariamente. Assim, entende-se que os critérios definidos devem ser estritamente particulares para a problemática estudada.

Buscou-se primeiramente realizar uma coleta daqueles critérios recorrentemente citados em trabalhos acadêmicos publicados. Para isto foi realizada uma pesquisa em artigos de revistas na base de dados da Scopus para o período entre 2000 e 2020. Justifica-se apenas a base de dados escolhida por ser a que abrange a maior quantidade de publicações no tema, e por contemplar revistas de grande peso na área de patrimônio (e.g. Journal of Cultural Heritage), e o período pelos poucos resultados satisfatórios antes da data estabelecida. Ainda se observou uma altíssima quantidade de publicações duplicadas quando utilizada outras bases de dados (e.g. Web of Science \& Engineering Village) na etapa de definição de protocolo, e pela inexistência de artigos que contribuiriam no Periódicos da Capes.

Com os critérios e subcritérios levantados, optou-se por classificá-los devido a grande quantidade de termos usados nas publicações. Isto foi feito para permitir visualizar com facilidade como as pesquisas estavam lidando com as disciplinas que envolvem o processo decisório. De forma a representar a frequência dos critérios que foram classificados, como também considerando o peso que estes critérios obtiveram nos resultados das publicações, as classes foram pontuadas de 1 a 3 pontos. Onde 1 ponto significa que a classe tem poucos critérios representados naquele artigo e com pesos baixos, para 2 pontos podem ter alguns critérios com peso médio, enquanto que para 3 ela é representada por critérios com alto peso.

Tabela 1 - Revisão de critérios e temas em MCDM em patrimônio histórico edificado

\begin{tabular}{|c|c|c|c|c|c|c|c|}
\hline Publicações \Critérios & Tema Amplo & U & 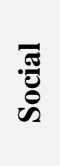 & 苞 & 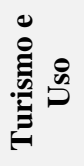 & 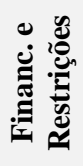 & 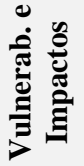 \\
\hline Ribera, Nesticò e Cucco (2019) & Conservação & $\cdots$ & $\bullet$ & • & $\ddot{\bullet}$ & $\bullet$ & • \\
\hline Ighravwe e Oke (2019) & Manutenção & & 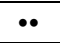 & & $\bullet$ & 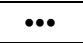 & $\bullet \bullet$ \\
\hline Nesticò e Somma (2019) & Conservação & $\cdots$ & $\bullet$ & - & $\bullet$ & $\ddot{\bullet \cdot}$ & $\cdot$ \\
\hline Falcão et al (2019) & Requalificação & $\bullet$ & $\cdot$ & $\cdot$ & $\bullet$ & $\cdot$ & $\cdot$ \\
\hline Prieto, Macías-Bernal e Silva (2019) & Conservação & $\bullet$ & $\bullet$ & & $\bullet$ & 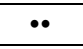 & 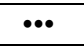 \\
\hline Fatoric e Seekamp (2018) & Conservação & 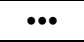 & & & 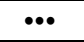 & & \\
\hline Nesticò, Morano e Sica (2018) & Requalificação & & & & 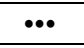 & $\cdots$ & $\cdots$ \\
\hline Turskis, Morkunaite e Kutut (2017) & Conservação & $\bullet$ & $\bullet$ & $\cdots$ & •.• & 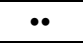 & \\
\hline Pinero et al (2017) & Requalificação & $\bullet$ & $\bullet$ & & $\cdot$ & 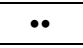 & $\cdots$ \\
\hline Jajac, Rogulj, Radnic (2017) & Requalificação & $\bullet$ & & & $\bullet$ & $\cdots$ & $\bullet$ \\
\hline Oppio (2015) & Avaliação & & $\bullet$ & & 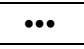 & 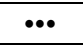 & $\bullet$ \\
\hline Ferretti, Bottero e Mondini (2014) & Requalificação & & $\bullet$ & & $\cdots$ & $\cdot$ & \\
\hline Vodopivec (2014) & Requalificação & $\bullet$ & $\bullet$ & $\bullet$ & $\bullet$ & $\cdots$ & $\cdots$ \\
\hline Ortiz et al (2014) & Avaliação & & $\cdot$ & & $\cdot$ & & $\cdots$ \\
\hline Kutut, Zavadskas e Lazauskas (2013) & Conservação & $\cdots$ & $\bullet$ & $\bullet$ & & & \\
\hline Turskis, Zavadskas e Kutut (2013) & Avaliação & $\cdots$ & & $\bullet$ & $\cdot$ & & \\
\hline Wang e Zeng (2010) & Requalificação & $\bullet$ & 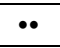 & $\ddot{\bullet}$ & $\cdot$ & $\bullet$ & $\bullet$ \\
\hline Yau (2009) & Conservação & $\cdot$ & $\cdot$ & & & $\cdots$ & $\cdots$ \\
\hline Dutta e Husain (2009) & Conservação & $\bullet$ & $\bullet$ & $\bullet$ & $\bullet$ & $\bullet$ & \\
\hline Bana e Costa (2002) & Requalificação & $\cdots$ & $\cdots$ & & - & & $\bullet$ \\
\hline
\end{tabular}


Fundamento nos artigos encontrados na revisão, de trabalhos acadêmicos como teses e dissertações sobre o caso, da análise de documentos que incluem o estudo de tombamento, o planejamento e a preservação do patrimônio edificado de Sobral, e com a consulta a profissionais envolvidos com a temática, a seguir se apresenta os critérios para representação do processo decisório de manutenção preventiva para as alternativas levantadas acompanhadas de sua justificativa encontradas na Tabela 2.

Tabela 2 - Critérios para Estudo do Caso de Sobral Vulnerabilidade Estrutural (VUL)

As estruturas do patrimônio arquitetônico, por sua própria natureza e história, apresentam uma série de desafios em diagnóstico e restauração que limitam a aplicação de códigos legais e padrões de construção (ICOMOS, 2003), sendo conservadas buscando ampliar sua vida (IPHAN, 2010). Este critério será traduzido em subcritérios buscando atender a metodologia de Vicente (2008), adotada em parte por Mota, Gadelha e Mesquita (2017) na cidade Sobral.

\begin{tabular}{|c|c|}
\hline 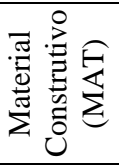 & $\begin{array}{l}\text { As estruturas dos edificados históricos são usualmente construídas com técnicas e materiais adotados na } \\
\text { época a qual foram concebidas, com baixo controle e reduzida qualidade. As edificações de Sobral atendem } \\
\text { a esta descrição por serem, em sua maioria, edificações de arquitetura vernacular, e que possuem como } \\
\text { principal elemento blocos cerâmicos (MOTA; GADELHA; MESQUITA, 2017). }\end{array}$ \\
\hline 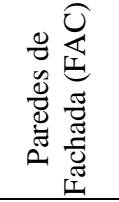 & $\begin{array}{l}\text { paredes com maior espessu } \\
\text { berta. As fachadas dos edifíc }\end{array}$ \\
\hline 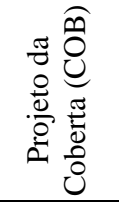 & $\begin{array}{l}\text { As cobertas influem na estabilidade estrutural pois, quando n } \\
\text { da esbeltes e condição de vinculação das peças entre si, cont } \\
\text { com ligações aos elementos estruturais encontrados nos perín } \\
\text { madeira, quando não apropriadamente preservados, oferec } \\
\text { VERSTRYNGE; VAN BALEN, 2018) }\end{array}$ \\
\hline
\end{tabular}
Significância Cultural (SIG)

Ao contrário da manutenção em edifícios comuns, esta operação em edifícios históricos deve sempre levar em consideração o valor do monumento, bem como as características da estrutura (PETZET, 2004). o ambiente em que se encontra, deste modo, os estudos de tombamento, bem como os de preservação Os patrimônios arquitetônicos se diferenciam das outras edificações principalmente neste valor, seja este tangível ou intangível (VECCO, 2010).

\begin{tabular}{|c|c|}
\hline 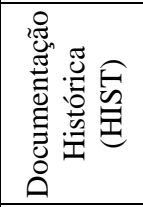 & $\begin{array}{l}\text { De acordo com Sant Anna (1996) a justificativa do tombamento de Sobral partiu, de forma inédita de } \\
\text { parâmetros que evidenciam o conceito de Cidade-Documento, e Duarte Junior (2012) complementa que nos } \\
\text { processos de tombamento, o sítio histórico de Sobral foi abordado parte como documento ao se considerar a } \\
\text { contribuição histórica para a constituição da sede municipal e a estruturação da região, mediante a valorização } \\
\text { filológica da maneira como se deu sua formação e evolução histórica, sócio-econômica e urbana. }\end{array}$ \\
\hline 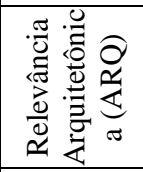 & $\begin{array}{l}\text { onumento, a } \\
\text { rquitetônico, } \\
\text { tico na fusão }\end{array}$ \\
\hline & $\begin{array}{l}\text { Em Sobral, dentre os objetivos que cara } \\
\text { manutenção de laços dos bens culturais com } \\
\text { a manutenção das importantes funções so }\end{array}$ \\
\hline
\end{tabular}

Potenciais e Restrições (POT)

Pelo seu valor tangível e intangível os patrimônios edificados, principalmente quando estrategicamente requalificados, podem oferecer uma oportunidade não só de integração social e bem-estar comunitário, como também valorizarem a região. Isto influi na valorização imobiliária, na captação de investimentos, no turismo e no comércio. Ainda, este potencial é ampliado pela frequente boa localização dos monumentos arquitetônicos, que muitas vezes são destinados para fins diferentes daqueles de sua origem.

\begin{tabular}{l|l} 
O turismo é uma das atividades importantes para a economia das cidades, e que se torna ainda mais oportuno \\
quando sua administração permite a integração da comunidade e a valorização do patrimônio (UNESCO,
\end{tabular}


Tabela 2 - Critérios para Estudo do Caso de Sobral (cont)

\begin{tabular}{|c|c|}
\hline 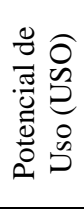 & $\begin{array}{l}\text { O uso e reuso dos patrimônios edificados é um tema extensivamente recorrente no meio acadêmico como } \\
\text { apontado na análise de Morkūnaitè, Kalibatas e Kalibatienè (2019). Positivamente, Duarte Junior (2012) } \\
\text { relata que em Sobral o tombamento permitiu a experimentação de novos usos com novas tipologias e } \\
\text { morfologias. Se observa frequentes iniciativas neste âmbito, não só do setor público, sendo a mais recente a } \\
\text { futura utilização da Estação Ferroviária de Sobral para abrigar a Escola de Artes Visuais. }\end{array}$ \\
\hline 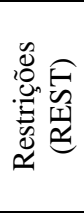 & $\begin{array}{l}\text { Reconhecendo a complexidade da manutenção nos edificados históricos apontadas como restrições e } \\
\text { dificuldades apresentadas em Gard'ner (2007) principalmente no que tange as normativas e políticas e suas } \\
\text { particularidades, os requisitos regulatórios e legislativos, a ausência de materiais e técnicas apropriadas para } \\
\text { as atividades de manutenção, e os múltiplos stakeholders envolvidos, este subcritério é importante para o } \\
\text { modelo avaliar os bens nestes aspectos. }\end{array}$ \\
\hline
\end{tabular}

\subsection{Ponderação de Critérios}

Seguindo a Etapa 2 do framework proposto (Figura 1), a ponderação dos critérios se inicia com a determinação de áreas chaves, relacionadas aos critérios selecionados. Para o estudo do caso de Sobral foram escolhidas cinco áreas: Engenharia, Arquitetura, Artes, História e Administração. A escolha das áreas se justifica na participação no processo decisório dessas disciplinas, principalmente quando se considera uma abordagem multicritério. Para cada uma das áreas foi escolhido apenas um profissional, este especialista foi apresentado ao estudo de caso através de uma breve exposição da problemática, de conceitos, e dos critérios. Os critérios foram consolidados através da confirmação de sua importância para a manutenção das alternativas. Em seguida foi feita a edução do conhecimento do especialista para realizar a análise par-a-par dos critérios. Para isto foi utilizado o recurso de formulários online, que pode ser consultado em https://forms.gle/CpjKpqFiPYYHd4GM6. Apesar do método BBW reduzir a quantidade de avaliações, a forma como se faz a comparação entre o melhor e o pior critério se mostrou não inteligível, pois ora se associa importância com valores menores, e ora com valores maiores. Assim, a criação do vetor de comparação entre os outros e o pior critério foi mantida, enquanto para o vetor de comparação entre o melhor e os outros foi feita da seguinte forma:

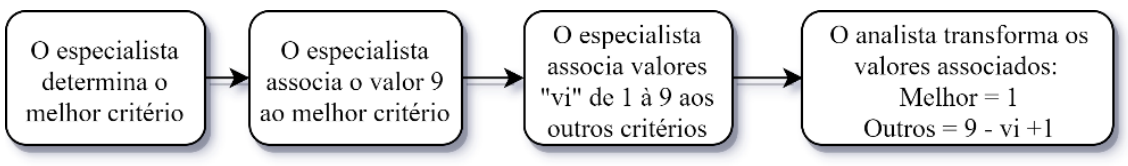

Figura 4: Modificação em coleta de vetor de melhor com outros

Fica evidente que a transformação foi feita para não mudar as operações posteriores do método. Os resultados da edução do conhecimento dos especialistas, e da transformação dos vetores de melhor com os outros é apresentada na Tabela 3.

Tabela 3 - Revisão de critérios e temas em MCDM em patrimônio histórico edificado

\begin{tabular}{|c|c|c|c|c|c|c|c|c|c|c|c|c|c|c|c|c|}
\hline \multirow[t]{2}{*}{ Esp. } & \multicolumn{8}{|c|}{ Critérios } & \multicolumn{8}{|c|}{ Vulnerabilidade Estrutural } \\
\hline & BEST & VUL & SIG & POT & WORST & VUL & SIG & POT & BEST & MAT & FAC & $\mathrm{COB}$ & WORST & MAT & FAC & $\mathrm{COB}$ \\
\hline Eng. & VUL & 1 & 2 & 4 & POT & 4 & 3 & 1 & FAC & 4 & 1 & 3 & MAT & 1 & 4 & 3 \\
\hline Arq. & SIG & 3 & 1 & 5 & POT & 3 & 5 & 1 & FAC & 4 & 1 & 1 & MAT & 1 & 3 & 4 \\
\hline Art. & SIG & 1 & 1 & 3 & POT & 4 & 4 & 1 & FAC & 2 & 1 & 2 & $\mathrm{COB}$ & 2 & 3 & 1 \\
\hline Hist. & SIG & 1 & 1 & 4 & POT & 4 & 5 & 1 & MAT & 1 & 3 & 1 & FAC & 3 & 1 & 4 \\
\hline \multirow[t]{3}{*}{ Adm. } & VUL & 1 & 3 & 2 & SIG & 4 & 1 & 2 & FAC & 3 & 1 & 3 & $\mathrm{COB}$ & 2 & 4 & 1 \\
\hline & \multicolumn{8}{|c|}{ Significância Cultural } & \multicolumn{8}{|c|}{ Potenciais e Restrições } \\
\hline & BEST & HIST & SOC & ARQ & WORST & HIST & SOC & ARQ & BEST & TUR & USO & REST & WORST & TUR & USO & REST \\
\hline Eng. & SOC & 4 & 1 & 6 & ARQ & 5 & 5 & 1 & USO & 4 & 1 & 2 & TUR & 1 & 5 & 4 \\
\hline Arq. & SOC & 4 & 1 & 6 & ARQ & 2 & 4 & 1 & USO & 7 & 1 & 2 & TUR & 1 & 7 & 5 \\
\hline Art. & SOC & 1 & 1 & 3 & ARQ & 3 & 4 & 1 & USO & 2 & 1 & 1 & TUR & 1 & 4 & 3 \\
\hline Hist. & HIST & 1 & 1 & 3 & ARQ & 3 & 3 & 1 & TUR & 1 & 4 & 1 & USO & 5 & 1 & 4 \\
\hline Adm. & SOC & 2 & 1 & 5 & ARQ & 3 & 4 & 1 & REST & 5 & 2 & 1 & TUR & 1 & 3 & 5 \\
\hline
\end{tabular}

A Tabela 3 está dividida em quatro seções, uma para os critérios, e uma para cada um dos subcritérios. Na coluna "BEST" é apresentado o critério selecionado como melhor pelo especialista, enquanto na "WORST" o pior. A coleta de dados se 
deu de forma fácil e dinâmica, e o instrumento indica uma boa construção, visto a identificação de poucos valores extremos ou idênticos para o mesmo especialista, na verdade, constatou-se valores próximos entre os especialistas, o que satisfaz o método e torna o resultado mais significativo, mesmo não havendo ainda nenhum indicador para isto no método. Apesar da construção dos vetores de melhor e pior terem sido realizados em seções diferentes, isto feito de forma planejada para identificar avaliações discrepantes, existe forte consistência, e todos os critérios ou subcritérios ora identificados como pouco importantes na primeira fase, foram indicados como o pior critério na segunda fase. De forma análoga para os critérios mais importantes na primeira e segunda fase. A Figura 5 apresenta a árvore de critérios, com os pesos associados a eles e seus subcritérios, e o peso global dos subcritérios em negrito. Na Tabela se apresenta a ordem de importância dos subcritérios.

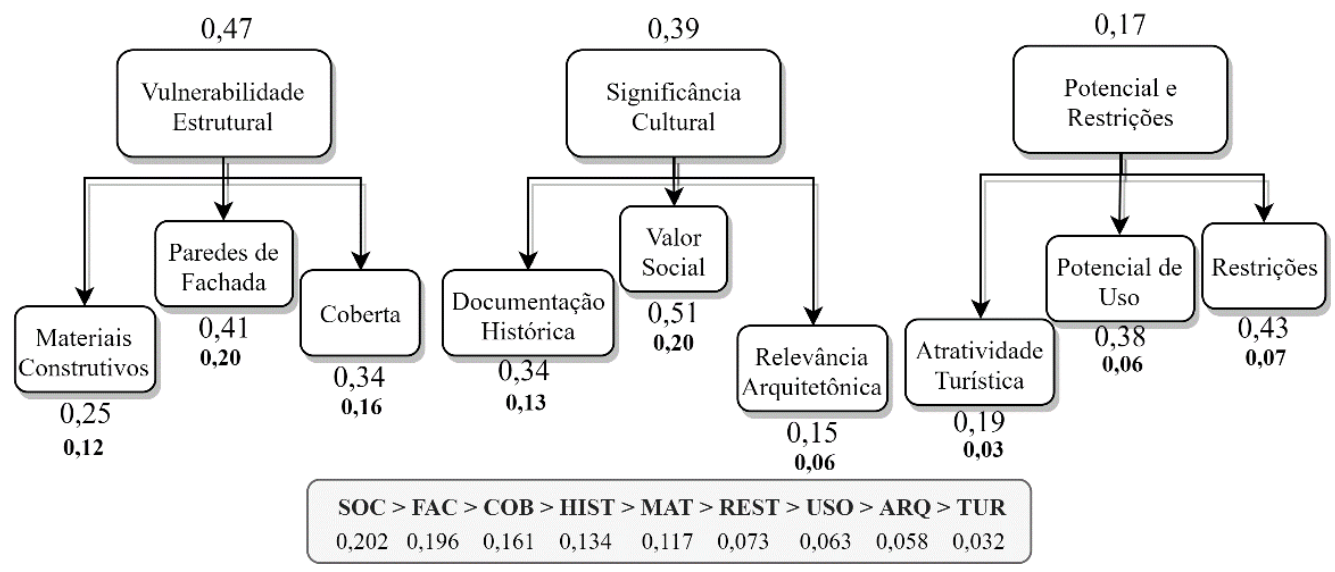

Figura 5: Árvore de critérios e pesos

Os pesos representam a importância de cada um dos critérios para a problemática levantada, assim, só pode se dizer que a vulnerabilidade estrutural é mais importante que a significância cultural de um patrimônio arquitetônico no que compreende as atividades de manutenção preventiva. Os resultados permitem construir uma ordem hierárquica dos critérios e subcritérios, assim a vulnerabilidade se mostra sensivelmente mais importante que significância cultural para manutenção preventiva. Certamente essa diferença seria maior caso as manutenções compreendessem atividades de urgência devido ameaça à perda do patrimônio. O critério de potencial e restrições obteve um reduzido valor em comparação aos outros dois critérios. De fato, com exceção do profissional de administração, todos os outros especialistas consideraram este critério como o menos importante. É possível neste momento compreender a sensibilidade do modelo, que mostrou resultados muito alinhados com os apresentados entre os decisores, isto fica mais claro devido a não atribuição de pesos aos próprios especialistas. Se optou por não realizar esta prática, pois não existe hierarquia entre os especialistas, não se desejando privilegiar áreas, e sim contribuir para o contexto de decisão multidisciplinar para o objeto de estudo. Esta contribuição se torna mais interessante quando se observa que os especialistas não privilegiam suas áreas de estudo, entendendo-se com isto que a problemática foi absorvida por eles e analisada de forma racional.

O subcritério que recebeu maior importância foi o aspecto social, seguido por paredes de fachada, cobertas, e documentação histórica. Observa-se na Tabela 1 a frequência com que o aspecto social foi incluso, bem como considerado relevante. Isto acontece em múltiplas publicações, mesmo naquelas com supostamente menos envolvimento da comunidade. Mesmo no caso de Sobral, que se limita a manutenção almejando a preservação dos bens, este subcritério se mantém em primeiro lugar. Conclui-se que a percepção dos especialistas consultados está alinhada com a integração da comunidade ao patrimônio como instruído em IPHAN (2018), bem como ambos consentem com a comunidade acadêmica internacional que normalmente consideram os documentos e debates propostos pela UNESCO e o ICOMOS. Se verifica nestes resultados a maior importância das fachadas sob a coberta e materiais construtivos, alinhando-se a avaliação de Vicente (2008) quanto a vulnerabilidade sísmica. Não só pelo aspecto estrutural, os resultados quanto a importância da fachada também pode ter relação ao valor estético e morfológico da cidade.

A atratividade turística foi considerada o subcritério menos importante relevante a priorização da manutenção preventiva do patrimônio. Pode-se justificar isto pelo entendimento dos especialistas pelo pouco potencial turístico das alternativas levantadas, mesmo no que se refere ao turismo religioso, já em Turskis, Morkunaite e Kutut (2017) este subcritério tem forte relevância devido as características das edificações. O segundo menos importante é a relevância arquitetônica. Apesar de parecer controverso, a disciplina se mostrou com pouco peso dentre alguns estudos apresentados na Tabela 1, 
mesmo no que tange a conservação destes bens como em Yau (2009), como na requalificação para reuso em Ribera et al. (2019). O próprio estudo de tombamento compreende que valor histórico destas edificações perpassam a estética arquitetônica, apesar de estarem fortemente relacionadas. Mesmo na Tabela 2 pode-se verificar que o especialista da área de arquitetura julgou o subcritério como relativamente pouco importante para a manutenção das alternativas. Estas informações devem ser um ponto de partida para abrir um debate proveitoso sobre o tema, que em alguns cenários se restringe aos arquitetos. Mesmo que por força de lei eles sejam os profissionais responsáveis por projetos de restauração de bens arquitetônicos, e normalmente estão envolvidos mais ativamente nas principais decisões que abrangem estas edificações, é necessário incluir as disciplinas relacionadas com os problemas específicos, principalmente quando abrange o planejamento estratégico da cidade.

A Figura 6 apresenta graficamente o Credal Ranking entre critérios e entre os seus subcritérios. O Credal Ranking avalia o grau de confiança de quanto um critério, ou subcritério, é superior a outro, variando de 0 (nenhuma confiança de superioridade) à 1 (total confiança de superioridade). As avaliações de confiança são realizadas entre pares, entretanto, como os critérios foram avaliados em conjuntos de três, a quantidade de comparações par-a-par são, também, apenas três. No gráfico, os nós representam os critérios, ou subcritérios, enquanto a seta de um nó a outro representa a direção da superioridade e o número encontrado sobre a seta representa a confiança desta superioridade. Assim, em (a) o critério SIG é superior ao critério POT com 0,99 de confiança, enquanto VUL é superior à SIG com 0,66 de confiança, o que pode ser considerada uma confiança baixa, dependendo da rigorosidade no processo decisivo esta superioridade poderia ser contestada.

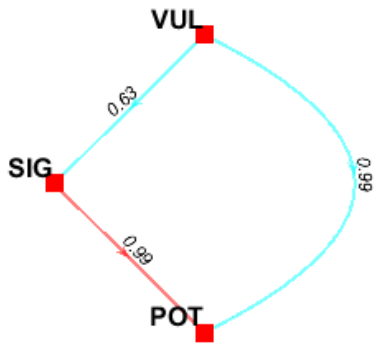

(a)

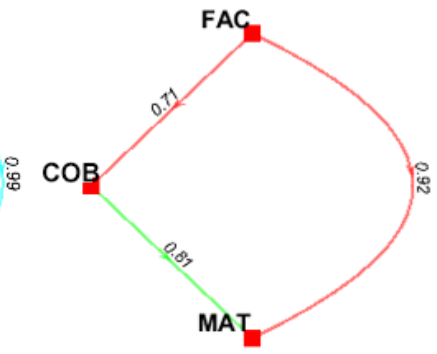

(b)

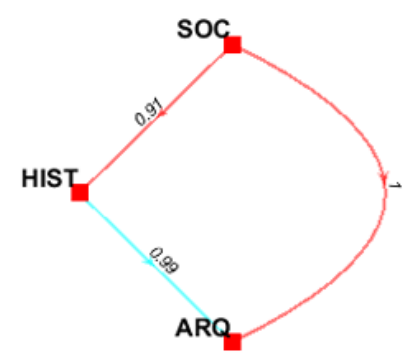

(c)

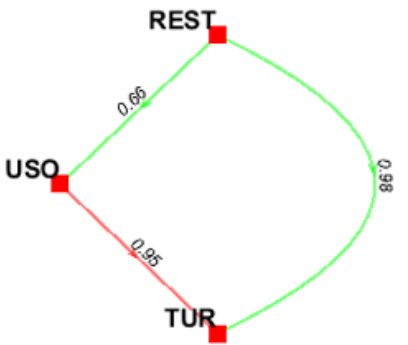

(d)

Figura 6: Credal Ranking (a) critérios (b) subcritérios de VUL (c) subcritérios de SIG (d) subcritérios de POT

Desta forma o Credal Ranking é uma ferramenta muito útil para o tomador de decisão, principalmente quando realizada em grupos, nutrindo um debate sobre a relação entre os critérios e subcritérios. Para Mohammadi e Rezaei (2019) ela se trata de uma informação ainda mais relevante em decisões onde os vetores representam as preferências do grupo. Para os resultados discutidos neste trabalho os graus de confiança atenderam às expectativas, onde ainda a confiança de superioridade se aproxima com a relação entre os pesos apresentados na Figura 6. As informações coletadas do Credal Ranking deverão ser oportunas para a fase posterior da pesquisa, que envolve decisores envolvidos com a problemática.

\section{CONCLUSÕES}

Nas décadas recentes existe um aumento no interesse em patrimônios edificados principalmente no contexto europeu, mas com iniciativas importantes em outras localidades, como na Ásia e na América do Norte. Este interesse é traduzido em produções acadêmicas, e posteriormente em ações no âmbito tanto público como privado, que vem gerando novas discussões e contagiando a temática globalmente. A proteção desse patrimônio é importante, mas por si só não garante o desenvolvimento sustentável, no que tange a perspectiva econômica e social. Portanto, é necessário garantir a inclusão do patrimônio arquitetônico nos programas de gestão e desenvolvimento, visando atender as necessidades da sociedade. A indicação de uma função dos múltiplos critérios importantes provém ao tomador de decisão dados indispensáveis para avaliar a intervenção correta.

De fato, o patrimônio possui um valor cultural, social e econômico capaz de gerar processos virtuosos para o desenvolvimento do território circundante. Para preservar esse patrimônio adequadamente, interromper os frequentes processos de degradação e promover atividades de recuperação, é necessário criar e promover ferramentas capazes de lidar com os múltiplos agentes e disciplinas. Essas ferramentas devem ser integradas ao planejamento no âmbito governamental, e sempre que oportuno, no jurídico, isto pois apenas com este apoio é possível implementar às ações necessárias à sua proteção, uma vez que o ambiente histórico é vulnerável. 
Entendendo a manutenção preventiva como parte das atividades de conservação, este trabalho propõe um framework que considera as características dos patrimônios edificados alinhados com uma visão multidisciplinar, que envolvem múltiplos agentes e critérios, presentes em um ambiente reconhecidamente complexo. Esta abordagem é apropriada considerando as recentes diretrizes do IPHAN (2018), que está alinhada com àquelas encontradas nos manuais de gestão do patrimônio material da UNESCO (2016), como também com os mais recentes debates relatados e propostos em publicações acadêmicas e nos eventos do ICOMOS. Para isto, o modelo adota dois métodos de decisão multicritério em duas etapas.

A primeira etapa, relatada neste trabalho, demonstra como aspectos multidisciplinares na manutenção preventiva nestas edificações pode ser tratado apropriadamente. Para isto, critérios foram identificados e consolidados com a consulta a documentos específicos ao estudo do caso, bem como a especialistas e acadêmicos que conhecem o cenário. Sobral é uma cidade a frente do seu tempo com relação a preservação do seu patrimônio edificado, com papel da comunidade e de ações governamentais municipais que reconheceram o valor o patrimônio através de um caminho de desenvolvimento sustentável. Desta forma, a ferramenta tem aplicabilidade e relevância por considerar a opinião de especialistas adequadamente, considerar a visão da administração pública, e promover o debate, pois ambos os métodos tem como análise de sensibilidade a avaliação dos resultados com informações adicionais relevantes.

\section{AGRADECIMENTOS}

O presente trabalho foi realizado com apoio da Coordenação de Aperfeiçoamento de Pessoal de Nível Superior - Brasil (CAPES) - Código de Financiamento 001

\section{REFERÊNCIAS}

BANA E COSTA, C. A.; OLIVEIRA, R. C. Assigning priorities for maintenance, repair and refurbishment in managing a municipal housing stock. European Journal of Operational Research, 2002.

BOND, S.; WORTHING, D. Managing Built Heritage: The role of cultural values and significance. Chichester, UK: Wiley-Blackwell, 2016. v.

BRASIL. Decreto Lei 25/1937, dispõe sobre proteção ao patrimônio histórico-cultural. Rio de Janeiro, RJ, 1937.

DUARTE JUNIOR, R. Sítios Históricos Brasileiros: Monumento, Documento, Empreendimento e Instrumento - O Caso de Sobral-CE. 2012. 460 f. Tese (Arquitetura)- Universidade Federal de São Paulo. Departamento de Arquitetura e Urbanismo. São Paulo, SP, Brasil, 2012.

DUTTA, M.; HUSAIN, Z. An application of Multicriteria Decision Making to built heritage. The case of Calcutta. Journal of Cultural Heritage, v. 10, n. 2, p. 237-243, 2009.

FAlCÃO, A. P.; MACHETE, R.; CASTILHO GOMES, M.; GONÇALVES, A. B. Spatial Multi-Criteria Decision Analysis for Rehabilitation Priority Ranking: A Collaborative Application to Heritage Workforce Housing Sites. International Journal of Architectural Heritage, v. 0, n. 0, p. 1-17, 2019.

FATORIĆ, S.; SEEKAMP, E. A measurement framework to increase transparency in historic preservation decisionmaking under changing climate conditions. Journal of Cultural Heritage, v. 30, p. 168-179, 2018.

FERRETTI, V.; BOTTERO, M.; MONDINI, G. Decision making and cultural heritage: An application of the MultiAttribute Value Theory for the reuse of historical buildings. Journal of Cultural Heritage, v. 15, n. 6, p. 644-655, 2014.

FORSYTH, M. Understanding historic building conservation. 1st. ed. Oxford: Blackwell Publishing, 2007.

GARD’NER, J. M. Preparing the conservation plan. In: Understanding historic building conservation. [s.l: s.n.]p. 156174.

ICOMOS. Principles for the analysis, conservation and structural restoration of architectural heritage. In: ICOMOS 14th General Assembly in Victoria Falls, Zimbabwe. Anais... Zimbabwe: 2003. 
IGHRAVWE, D. E.; OKE, S. A. A multi-criteria decision-making framework for selecting a suitable maintenance strategy for public buildings using sustainability criteria. Journal of Building Engineering, v. 24, n. March, p. 100753, 2019.

IPHAN. Estudo para tombamento do conjunto urbanístico da cidade de Sobral-Ceará - Volume 2, 1998.

IPHAN. Portaria $\mathbf{n}^{0}$ 420, de 22 de dezembro de 2010 - Procedimentos para realização de intervenções em bens edificados tombados e nas respectivas áreas de entorno, 2010.

IPHAN. Portaria No 375 de 19 de Setembro de 2018 - Política de Patrimônio Cultural Material, 2018. . Disponível em: <http://acesso.iphan.gov_br/cprod/termoAberturaj>.

JAJAC, N.; ROGULJ, K.; RADNIĆ, J. Selection of the Method for Rehabilitation of Historic Bridges-A Decision Support Concept for the Planning of Rehabilitation Projects. International Journal of Architectural Heritage, v. 11, n. 2, p. 261-277, 2017.

KUTUT, V.; ZAVADSKAS, E. K.; LAZAUSKAS, M. Assessment of priority options for preservation of historic city centre buildings using MCDM (ARAS). Procedia Engineering, v. 57, p. 657-661, 2013.

MOHAMMADI, M.; REZAEI, J. Bayesian best-worst method: A probabilistic group decision making model. Omega (United Kingdom), n. June, 2019.

MORKŪNAITÉ, Ž.; KALIBATAS, D.; KALIBATIENĖ, D. A bibliometric data analysis of multi-criteria decision making methods in heritage buildings. Journal of Civil Engineering and Management, v. 25, n. 2, p. 76-99, 2019.

MOTA, L.; GADELHA, A.; MESQUITA, E. Método simplificado de avaliação da vulnerabilidade estrutural de construções históricas Simplified method of assessing the structural vulnerability of historic buildings. n. September, p. 18, 2017.

NASCIMENTO, J. C. do. Re-descobrirão o Ceará? Representações dos sítios históricos de Icó e Sobral: entre areal e patrimônio nacional. Salvador, BA. EDUFBA, 2011

NESTICÒ, A.; MORANO, P.; SICA, F. A model to support the public administration decisions for the investments selection on historic buildings. Journal of Cultural Heritage, v. 33, n. 2018, p. 201-207, 2018.

NESTICÒ, A.; SOMMA, P. Comparative analysis of multi-criteria methods for the enhancement of historical buildings. Sustainability (Switzerland), v. 11, n. 17, 2019.

OPPIO, A.; BOTTERO, M.; FERRETTI, V.; FRATESI, U.; PONZINI, D.; PRACCHI, V. Giving space to multicriteria analysis for complex cultural heritage systems: The case of the castles in Valle D'Aosta Region, Italy. Journal of Cultural Heritage, v. 16, n. 6, p. 779-789, 2015.

ORTIZ, P.; ANTUNEZ, V.; MARÍA, J.; ORTIZ, R.; AUXILIADORA, M.; GALÁN, E. Approach to environmental risk analysis for the main monuments in a historical city. Journal of Cultural Heritage, v. 15, n. 4, p. 432-440, 2014.

PERNG, Y. H.; JUAN, Y. K.; HSU, H. S. Genetic algorithm-based decision support for the restoration budget allocation of historical buildings. Building and Environment, 2007.

PETZET, M. Principles of preservation: An introduction to the International Charters for Conservation and Restoration 40 years after the Venice Charter. International Charters for Conservation and Restoration. Monuments \& Sites, p. 7-29, 2004. Disponível em: <http://openarchive.icomos.org/432/1/Monuments_and_Sites_1_Charters_Petzet.pdf>.

PIÑERO, I.; SAN-JOSÉ, J. T.; RODRÍGUEZ, P.; LOSÁÑEZ, M. M. Multi-criteria decision-making for grading the rehabilitation of heritage sites. Application in the historic center of La Habana. Journal of Cultural Heritage, v. 26, p. 144-152, 2017.

PORTELA, M. L. B. Preservação e desenvolvimento: a dinãmica sócio-econômica do sítio histórico de Sobral/CE. 
2018. 220 f. Dissertação (Arquitetura) Universidade Federal do Ceará, Departamento de Arquitetura e Urbanimos, Fortaleza, CE, Brasil. 2018.

PRIETO, A. J.; MACÍAS-BERNAL, J. M.; SILVA, A.; ORTIZ, P. Fuzzy Decision-Support System for Safeguarding Tangible and Intangible Cultural Heritage. Sustainability, v. 11, n. 3953, p. 1-12, 2019.

REZAEI, J. Best-worst multi-criteria decision-making method. Omega (United Kingdom), v. 53, p. 49-57, 2015.

RIBERA, F.; NESTICÒ, A.; CUCCO, P.; MASELLI, G. A multicriteria approach to identify the Highest and Best Use for historical buildings. Journal of Cultural Heritage, 2019.

SANT ANNA, M. Termo de referencia para desenvolvimento dos estudos de tombamento em Sobral-CE. Sobral, IPHAN, 1996.

SOBRAL. Plano de Desenvolvimento Urbano Integrado. 2018. Disponível em: <http://www.pduisobral.com.br/documentos> Acesso em: 16 de out de 2019.

TURSKIS, Z.; MORKUNAITE, Z.; KUTUT, V. A hybrid multiple criteria evaluation method of ranking of cultural heritage structures for renovation projects. International Journal of Strategic Property Management, v. 21, n. 3, p. 318-329, 2017.

TURSKIS, Z.; ZAVADSKAS, E. K.; KUTUT, V. A model based on ARAS-G and AHP methods for multiple criteria prioritizing of heritage value. International Journal of Information Technology and Decision Making, v. 12, n. 1, p. 45-73, 2013.

UNESCO. Managing Tourism at World Heritage Sites: a practical manual for world heritage site managersa Practivc. UNESCO. 2002.

UNESCO. Gestão do patrimônio mundial cultural: Manual de referência. UNES Organização das Nações Unidas para a Educação a Ciência e a Cultura, Place de Fontenoy, França. 2016

VAN ROY, N.; VERSTRYNGE, E.; VAN BALEN, K. A preventive conservation approach for historical timber roof structures. Journal of Cultural Heritage Management and Sustainable Development, v. 8, n. 2, p. 82-94, 2018.

VECCO, M. A definition of cultural heritage: From the tangible to the intangible. Journal of Cultural Heritage, v. 11, n. 3, p. 321-324, 2010.

VICENTE, R. da S. Estratégias e metodologias para intervenções de reabilitação urbana: Avaliação da vulnerabilidade e do risco sísmico do edificado. 2008.

VODOPIVEC, B.; ŽARNIĆ, R.; TAMOŠAITIENĖ, J.; LAZAUSKAS, M.; ŠELIH, J. Renovation priority ranking by multi-criteria assessment of architectural heritage: the case of castles. International Journal of Strategic Property Management, n. March 2015, p. 37-41, 2015.

WANG, H.; ZENG, Z. Expert Systems with Applications A multi-objective decision-making process for reuse selection of historic buildings. Expert Systems With Applications, v. 37, n. 2, p. 1241-1249, 2010.

WARREN, J. Conservation of structure in historic buildings. Journal of Architectural Conservation, v. 10, n. 2, p. 3949, 2004.

YAU, Y. Multi-criteria decision making for urban built heritage conservation: Application of the analytic hierarchy process. Journal of Building Appraisal, v. 4, n. 3, p. 191-205, 2009.

ZADEH, L. a. Fuzzy sets. Information and Control, v. 8, n. 3, p. 338-353, 1965.

ZAVADSKAS, E. K.; TURSKIS, Z.; KILDIENĖ, S. State of Art Surveys of Overviews on Mcdm/Madm Methods. Technological and Economic Development of Economy, v. 20, n. 1, p. 165-179, 2014. 